\title{
Lukasiewicz Fuzzy Implication Operator on Pythagorean Fuzzy Tautological Matrices
}

\author{
D. Venkatesan \\ Assistant Professor of Mathematics \\ BIST, BIHER, Bharath University \\ Chennai, India \\ venkat2733327gmail.com
}

Abstract - In this paper, introduced Pythagorean fuzzy tautologial matrices and Pythagorean fuzzy cotautological matrices and some properties of Lukasiwicz implication operator over Pythagorean fuzzy tautologial matrices and Pythagorean fuzzy cotautological matrices are discussed. Also discussed the relation between implication with Lukasiewicz disjunction and conjunction operations of PFCMs and PFCTMs.

Keywords- Intuitionistic Fuzzy Matrix, Pythagorean Fuzzy Set, Pythagorean Fuzzy Matrix, Disjunction, Conjunction, Implication.

\section{INTRODUCTION}

Pal et.al[3,10] introduced Intuitionistic fuzzy matrices also in[2], they discussed new operations on intuitionistic fuzzy matrices and investigated their algebraic properties. Atanassov and Tcvetkov[1] defined on Lukasiewicz's intuitionistic fuzzy disjunction and conjunction operations and investigated their algebraic properties. Muthuraji and Sriram[6] introduced two operators conjunction and disjunction from Lukasiewic'z type over intuitionistic fuzzy matrix(IFM) and investigated their algebraic properties. Also in [6], they proved the set of all IFMs is a commutative monoid under these operations.

Yager[15] introduced Pythagorean fuzzy set(PFS) characterized by a membership degree and a non membership degree satisfying the condition that the square sum of its membership degree and non membership degree is equal to or less than 1 , has much stronger ability than intuitionistic fuzzy set to model such uncertain information in multi-criteria decision making(MCDM) problems.

Zhang and $\mathrm{Xu}[16]$ defined some novel operational laws of PFS and discuss its desirable properties. The motivation of introducing PFSs is that in the real-life decision process, the sum of the support degree and the against degree to which an alternative satisfying a criterion provided by the decision maker may be bigger than 1 but their square sum is equal to or less than 1.

Silambarasan and Sriram[11] introduced Pythagorean fuzzy matrix(PFM) and its algebraic operations. Venkatesan and Sriram[12] they defined multiplicative operations of Pythagorean fuzzy matrices and studied some of the basic properties of these operations with other predefined operators. Muthuraji[7, 8] studied some properties of operations conjunction, disjunction and implication from Lukasiewicz's type over Intuitionistic fuzzy matrices. Venkatesn and sriram $[13,14]$ extend these operations to PFMs and studied some of the basic properties of these operations with other predefined operators.

Pal and Khan[4] introduced intuitionistic fuzzy tautological matrices and its algebraic operations. Murugadas and Lalitha[5] they defined intuitionistic fuzzy cotautological matrices and its algebraic operations.

Muthuraji and Anitha[9] they discused the Lukasiewicz imlication on intuitionistic fuzzy tautological matrices discussed its desirable properties. I extend these operations to PFMs and studied some of the basic properties of these operations with other predefined operators.

The remainder of this paper is organized as follows. In Section 2, the basic definitions of PFM are given. In Section 3, introduced Pythagorean fuzzy tautologial matrices and Pythagorean fuzzy cotautological matrices and some properties of Lukasiwicz implication operator over Pythagorean fuzzy tautologial matrices and Pythagorean fuzzy cotautological matrices are discussed.

\section{PRELIMINARIES}

In this section, some basic definitions which are essential for the development of this dissertation are discussed.

Definition 2.1. [3] A intuitionistic fuzzy matrix(IFM) is a matrix of pairs $A=\left(\left\langle a_{i j}, a_{i j}^{\prime}\right\rangle\right)$ of non negative real numbers $a_{i j}, a_{i j}^{\prime} \in[0,1]$ satisfying the condition $0 \leq a_{i j}+a_{i j}^{\prime} \leq 1$, for all $i, j$.

Definition 2.2. [2] For any two IFMs $A$ and $B$ of the same size, then 
$A \rightarrow_{1} B=\left(\left\langle\max \left(a_{i j}^{\prime}, b_{i j}\right), \min \left(a_{i j}, b_{i j}^{\prime}\right)\right\rangle\right)$.

Definition 2.3. [15] Let a set $X$ be a universe of discourse $A$ Pythagorean fuzzy set(PFS) $P$ is an object having the form, $P=\left(\left\langle x, P\left(\mu_{p}(x), v_{p}(x)\right) \mid(x \in X)\right\rangle\right)$, where the function $\mu_{p}: X \rightarrow[0,1]$ and $v_{p}: X \rightarrow[0,1]$ defines the degree of membership and degree of non-membership of the element $x \in X$ to $P$, respectively, and for every $x \in X$, it holds that $\left(\mu_{p}(x)\right)^{2}+\left(v_{p}(x)\right)^{2} \leq 1$.

Definition 2.4. [11] A Pythagorean fuzzy matrix(PFM) is a matrix of pairs $A=\left(\left\langle a_{i j}, a_{i j}^{\prime}\right\rangle\right)$ of a non negative real numbers $a_{i j}, a_{i j}^{\prime} \in[0,1]$ satisfying the condition $a_{i j}^{2}+a_{i j}^{\prime 2} \leq 1$, for all $i, j$.

The numbers $a_{i j}$ and $a_{i j}^{\prime}$ denote the degree of membership and the degree of non-membership of the $i j^{\text {th }}$ element in $A$ respectively.

Definition 2.5. [13] For any two PFMs $A, B \in \mathbb{P}_{m n}$, we have (i) $A^{C}=\left(\left\langle a_{i j}^{\prime}, a_{i j}\right\rangle\right)$ (The complement of $A$ ),

(ii) $A^{T}=\left(\left\langle a_{j i}, a_{j i}^{\prime}\right\rangle\right)$ (The transpose of $A$ ),

(iii) $A \leq B$ if and only if $a_{i j} \leq b_{i j}$ and $a_{i j}^{\prime} \geq b_{i j}^{\prime}$,

(iv) $A \geq B$ if and only if $a_{i j} \geq b_{i j}$ and $a_{i j}^{\prime} \leq b_{i j}^{\prime}$,

(iv) $A \wedge B=\left(\left\langle\min \left(a_{i j}, b_{i j}\right), \max \left(a_{i j}^{\prime}, b_{i j}^{\prime}\right)\right\rangle\right)$,

(v) $A \vee B=\left(\left\langle\max \left(a_{i j}, b_{i j}\right), \min \left(a_{i j}^{\prime}, b_{i j}^{\prime}\right)\right\rangle\right)$,

(vi) $\square A=\left(\left\langle a_{i j}, \sqrt{1-a_{i j}^{2}}\right\rangle\right)$,

(vii) $\diamond A=\left(\left|\sqrt{1-a_{i j}^{\prime}{ }^{2}}, a_{i j}^{\prime}\right|\right)$,

(viii) The $m \times n$ zero PFM $O$ is an PFM all of whose entries are $\langle 0,1\rangle$,

The $m \times n$ universal PFM $J$ is an PFM all of whose entries are $\langle 1,0\rangle$.

Definition 2.6. [13] For any two PFMs $A, B \in \mathbb{P}_{m n}$, we have (i) $A \vee_{L} B=$

$$
\left(\left|\sqrt{\min \left(1, a_{i j}^{2}+b_{i j}^{2}\right)}, \sqrt{\max \left(0, a_{i j}^{\prime 2}+{b_{i j}^{\prime}}^{2}-1\right)}\right|\right),
$$

(ii) $A \wedge_{L} B=$

$\left(\left(\sqrt{\max \left(0, a_{i j}^{2}+b_{i j}^{2}-1\right)}, \sqrt{\min \left(1, a_{i j}^{\prime 2}+b_{i j}^{\prime 2}\right)}\right\rangle\right)$.

Definition 2.7. [14] For any two PFMs $A, B \in \mathbb{P}_{m n}$, we have (i) $A \rightarrow_{L} B=$

$$
\left(\left|\sqrt{\min \left(1, a_{i j}^{\prime 2}+b_{i j}^{2}\right)}, \sqrt{\max \left(0, a_{i j}^{2}+b_{i j}^{\prime 2}-1\right)}\right\rangle\right),
$$

\section{MAIN RESULTS}

In this section, define Pythagorean Fuzzy Tautological Matrix (PFTM) and Pythagorean Fuzzy Cotautological Matrix (PFCTM) and some properties are conjunction, disjunction and implication are discussed over PFTM and have shown that some expressions involving all the above said operators always an PFCTM.

Definition 3.1. An Pythogorean fuzzy matrix of order $m \times n$ is called Pythagorean fuzzy tautological matrix(PFTM) if and only if $a_{i j} \geq a_{i j}^{\prime}$ for all $i, j$.

Definition 3.2. An Pythagorean fuzzy matrix of order $m \times n$ is called Pythogorean fuzzy cotautological matrix(PFCTM) if and only if $a_{i j} \leq a_{i j}^{\prime}$ for all $i, j$.

Property 3.1. For any two PFTMs $A, B \in \mathrm{P}_{m n}$, we have

(i) $A \vee_{L} B$ is an PFTM.

(ii) $A \wedge_{L} B$ is an PFM.

(iii) $A \rightarrow_{L} B$ is also an PFTM.

Proof:

From Definition 2.6,

$$
\begin{aligned}
& A \vee_{L} B \\
& =\left(\left|\sqrt{\min \left(1, a_{i j}^{2}+b_{i j}^{2}\right)}, \sqrt{\max \left(0, a_{i j}^{\prime 2}+{b_{i j}^{\prime}}^{2}-1\right)}\right|\right) .
\end{aligned}
$$

Since $\mathrm{A}$ and $\mathrm{B}$ are PFTMs.

From Definition 2.5, $a_{i j} \geq a_{i j}^{\prime}$ and $b_{i j} \geq b_{i j}^{\prime}$ then

$$
\sqrt{\min \left(1, a_{i j}^{2}+b_{i j}^{2}\right)} \geq \sqrt{\max \left(0, a_{i j}^{\prime 2}+b_{i j}^{\prime 2}-1\right)}
$$

Hence $A \vee_{L} B$ is an PFTM.

(ii) $A \wedge_{L} B=$

$$
\left(\left|\sqrt{\max \left(0, a_{i j}^{2}+b_{i j}^{2}-1\right)}, \sqrt{\min \left(1, a_{i j}^{\prime 2}+b_{i j}^{\prime 2}\right)}\right|\right) .
$$

Since $a_{i j} \geq a_{i j}^{\prime}$,

$$
\begin{aligned}
& \sqrt{\max \left(0, a_{i j}^{2}+b_{i j}^{2}-1\right)} \geq \sqrt{\min \left(1, a_{i j}^{\prime}{ }^{2}+b_{i j}^{\prime 2}\right)} \text { or } \\
& \sqrt{\max \left(0, a_{i j}^{2}+b_{i j}^{2}-1\right)} \leq \sqrt{\min \left(1, a_{i j}^{\prime 2}+b_{i j}^{\prime 2}\right)} \text {. }
\end{aligned}
$$

Hence $A \wedge_{L} B$ is an PFM.

(iii) $A \rightarrow_{L} B=$

$$
\left(\left|\sqrt{\min \left(1, a_{i j}^{\prime 2}+b_{i j}^{2}\right)}, \sqrt{\max \left(0, a_{i j}^{2}+b_{i j}^{\prime 2}-1\right)}\right|\right) .
$$

Since $b_{i j} \geq b_{i j}^{\prime}$, $\sqrt{\min \left(1, a_{i j}^{\prime 2}+b_{i j}^{2}\right)} \geq \sqrt{\max \left(0, a_{i j}^{2}+b_{i j}^{\prime 2}-1\right)}$.

Hence $A \rightarrow_{L} B$ is an PFTM.

Property 3.2. For any two PFCTMs $A, B \in \mathrm{P}_{m n}$, we have

(i) $A \vee_{L} B$ is an PFM.

(ii) $A \wedge_{L} B$ is an PFCTM.

(iii) $A \rightarrow_{L} B$ is also an PFTM. 


\section{Proof:}

From Definition 2.6,

$$
\begin{aligned}
& A \vee_{L} B \\
& =\left(\left|\sqrt{\min \left(1, a_{i j}^{2}+b_{i j}^{2}\right)}, \sqrt{\max \left(0,{a_{i j}^{\prime}}^{2}+b_{i j}^{\prime 2}-1\right)}\right|\right) .
\end{aligned}
$$

Since A and B are PFCTMs.

From Definition 2.5, $a_{i j} \leq a_{i j}^{\prime}$ and $b_{i j} \leq b_{i j}^{\prime}$ then $\sqrt{\min \left(1, a_{i j}^{2}+b_{i j}^{2}\right)} \leq \sqrt{\max \left(0, a_{i j}^{\prime 2}+b_{i j}^{\prime 2}-1\right)}$ or $\sqrt{\min \left(1, a_{i j}^{2}+b_{i j}^{2}\right)} \geq \sqrt{\max \left(0, a_{i j}^{\prime 2}+b_{i j}^{\prime 2}-1\right)}$.

Hence $A \vee_{L} B$ is an PFM.

(ii) $A \wedge_{L} B=$

$$
\left(\left|\sqrt{\max \left(0, a_{i j}^{2}+b_{i j}^{2}-1\right)}, \sqrt{\min \left(1,{a_{i j}^{\prime}}^{2}+{b_{i j}^{\prime}}^{2}\right)}\right|\right) .
$$

Since $a_{i j} \leq a_{i j}^{\prime}$,

$\sqrt{\max \left(0, a_{i j}^{2}+b_{i j}^{2}-1\right)} \leq \sqrt{\min \left(1, a_{i j}^{\prime 2}+b_{i j}^{\prime 2}\right)}$.

Hence $A \wedge_{L} B$ is an PFCTM.

(iii) $A \rightarrow_{L} B=$

$$
\left(\left|\sqrt{\min \left(1, a_{i j}^{\prime 2}+b_{i j}^{2}\right)}, \sqrt{\max \left(0, a_{i j}^{2}+b_{i j}^{\prime 2}-1\right)}\right\rangle\right) .
$$

Since $b_{i j} \leq b_{i j}^{\prime}$,

$\sqrt{\min \left(1, a_{i j}^{\prime 2}+b_{i j}^{2}\right)} \leq \sqrt{\max \left(0, a_{i j}^{2}+b_{i j}^{\prime 2}-1\right)}$.

Hence $A \rightarrow_{L} B$ is an PFCTM.

Property 3.3. For any A be an PFTM and B be an PFCTM

$A, B \in \mathrm{P}_{m n}$, we have

(i) $A \vee_{L} B$ is an PFTM.

(ii) $A \wedge_{L} B$ is an PFCTM.

(iii) $A \rightarrow_{L} B$ is also an PFM.

Proof:

(i) From Definition 2.6,

$$
\begin{aligned}
& A \vee_{L} B \\
& =\left(\left|\sqrt{\min \left(1, a_{i j}^{2}+b_{i j}^{2}\right)}, \sqrt{\max \left(0,{a_{i j}^{\prime}}^{2}+{b_{i j}^{\prime}}^{2}-1\right)}\right|\right) .
\end{aligned}
$$

From Definition 2.6, $a_{i j} \geq a_{i j}^{\prime}$ and $b_{i j} \geq b_{i j}^{\prime}$ then $\sqrt{\min \left(1, a_{i j}^{2}+b_{i j}^{2}\right)} \geq \sqrt{\max \left(0, a_{i j}^{\prime 2}+b_{i j}^{\prime 2}-1\right)}$.

Hence $A \vee_{L} B$ is an PFTM.

$$
\left(\left(\sqrt{\max \left(0, a_{i j}^{2}+b_{i j}^{2}-1\right)}, \sqrt{\min \left(1,{a_{i j}^{\prime}}^{2}+{b_{i j}^{\prime}}^{2}\right)}\right\rangle\right) .
$$

Since $a_{i j} \leq a_{i j}^{\prime}$,

$\sqrt{\max \left(0, a_{i j}^{2}+b_{i j}^{2}-1\right)} \leq \sqrt{\min \left(1, a_{i j}^{\prime 2}+b_{i j}^{\prime 2}\right)}$.

Hence $A \wedge_{L} B$ is an PFCTM.

(iii) $A \rightarrow_{L} B=$

$$
\left(\left|\sqrt{\min \left(1, a_{i j}^{\prime 2}+b_{i j}^{2}\right)}, \sqrt{\max \left(0, a_{i j}^{2}+{b_{i j}^{\prime}}^{2}-1\right)}\right|\right) .
$$

Since $a_{i j} \geq a_{i j}^{\prime}$ and $b_{i j} \leq b_{i j}^{\prime}$,

$\sqrt{\min \left(1, a_{i j}^{\prime 2}+b_{i j}^{2}\right)} \geq \sqrt{\max \left(0, a_{i j}^{2}+b_{i j}^{\prime 2}-1\right)}$ or

$\sqrt{\min \left(1, a_{i j}^{\prime 2}+b_{i j}^{2}\right)} \leq \sqrt{\max \left(0, a_{i j}^{2}+b_{i j}^{\prime 2}-1\right)}$.

Hence $A \rightarrow_{L} B$ is an PFM.

Corollary 3.1: Suppose A is an PFCTM and B is an PFTM then $A \rightarrow_{L} B$ is an PFTM.

Property 3.4. For any two PFMs $A, B \in \mathrm{P}_{m n}$, then the given statements are PFTMs.
(i) $A \rightarrow_{L} A$
(ii) $A \rightarrow_{L}\left(B \rightarrow_{L} A\right)$

Proof:

(i) $A \rightarrow_{L} A=$

$$
\left(\left|\sqrt{\min \left(1, a_{i j}^{\prime 2}+a_{i j}^{2}\right)}, \sqrt{\max \left(0, a_{i j}^{2}+{a_{i j}^{\prime}}^{2}-1\right)}\right|\right) .
$$

Now it is clear

$\sqrt{\min \left(1, a_{i j}^{\prime 2}+a_{i j}^{2}\right)} \geq \sqrt{\max \left(0, a_{i j}^{2}+a_{i j}^{\prime 2}-1\right)}$.

Hence $A \rightarrow_{L} A$ is an PFTM.

(ii) $B \rightarrow_{L} A=$

$$
\left(\left|\sqrt{\min \left(1, b_{i j}^{\prime 2}+a_{i j}^{2}\right)}, \sqrt{\max \left(0, b_{i j}^{2}+a_{i j}^{\prime 2}-1\right)}\right|\right) .
$$

$$
\begin{aligned}
& \left(\mid \sqrt{\min \left(1, \quad a_{i j}^{\prime 2}+b_{i j}^{\prime 2}+a_{i j}^{2}\right)},\right. \\
& \left.\sqrt{\max \left(0, a_{i j}^{2}+b_{i j}^{2}+a_{i j}^{\prime 2}-1-1\right)} \mid\right) .
\end{aligned}
$$

Since $\sqrt{\min \left(1,\left(a_{i j}^{\prime 2}+a_{i j}^{2}\right)+b_{i j}^{\prime 2}\right)} \geq$ $\sqrt{\max \left(0,\left(a_{i j}^{2}+{a_{i j}^{\prime}}^{2}\right)+b_{i j}^{2}-1-1\right)}$ for all $\mathrm{i}, \mathrm{j}$.

(ii) $A \wedge_{L} B=$ 


$$
\begin{aligned}
\sqrt{\min \left(1, \quad a_{i j}^{\prime 2}+b_{i j}^{\prime 2}+a_{i j}^{2}\right)} \\
\quad \geq \sqrt{\max \left(0, a_{i j}^{2}+b_{i j}^{2}+{a_{i j}^{\prime}}^{2}-1-1\right)}
\end{aligned}
$$

Suppose $\left(B \rightarrow_{L} A\right)=(1,0)$ for some $\mathrm{i}, \mathrm{j}$ then

$A \rightarrow_{L}\left(B \rightarrow_{L} A\right)$ is also $(1,0)$.

Hence $A \rightarrow_{L}\left(B \rightarrow_{L} A\right)$ is an PFTM.

Property 3.5. For any two PFMs $A, B \in \mathrm{P}_{m n}$, then the given statements are PFTMs.

(i) $A \rightarrow_{L}\left(A \vee_{L} B\right)$.

(ii) $B \rightarrow_{L}\left(A \vee_{L} B\right)$.

(iii) $A \rightarrow_{L}\left(B \rightarrow_{L}\left(A \vee_{L} B\right)\right)$.

Proof:

(i)

$$
\begin{aligned}
& A \vee_{L} B \\
& =\left(\left|\sqrt{\min \left(1, a_{i j}^{2}+b_{i j}^{2}\right)}, \sqrt{\max \left(0, a_{i j}^{\prime}{ }^{2}+b_{i j}^{\prime 2}-1\right)}\right|\right)
\end{aligned}
$$$$
A \rightarrow_{L}\left(A \vee_{L} B\right)
$$$$
=A \rightarrow_{L}\left(\left|\sqrt{\min \left(1, a_{i j}^{2}+b_{i j}^{2}\right)}, \sqrt{\max \left(0, a_{i j}^{\prime}{ }^{2}+b_{i j}^{\prime 2}-1\right)}\right\rangle\right)
$$

If $\sqrt{\min \left(1, a_{i j}^{2}+b_{i j}^{2}\right)}, \sqrt{\max \left(0, a_{i j}^{\prime 2}+b_{i j}^{\prime 2}-1\right)}=(1,0)$ then $A \rightarrow_{L}\left(A \vee_{L} B\right)=(1,0)$.

Otherwise

$$
\begin{aligned}
A \rightarrow_{L}\left(A \vee_{L} B\right)= & \left(\mid \sqrt{\min \left(1, a_{i j}^{\prime 2}+a_{i j}^{2}+b_{i j}^{2}\right)},\right. \\
& \left.\sqrt{\max \left(0, a_{i j}^{2}+{a_{i j}^{\prime}}^{2}+{b_{i j}^{\prime}}^{2}-1-1\right)} \mid\right) .
\end{aligned}
$$

It is clear that $\sqrt{\min \left(1, a_{i j}^{\prime 2}+a_{i j}^{2}+b_{i j}^{2}\right)} \geq$

$\sqrt{\max \left(0, a_{i j}^{2}+a_{i j}^{\prime 2}+b_{i j}^{\prime 2}-1-1\right)}$.

Hence $A \rightarrow_{L}\left(A \vee_{L} B\right)$ is an PFTM.

(ii) The proof of (ii) is similar to (i).

(iii) From (ii) $B \rightarrow_{L}\left(A \vee_{L} B\right)$ is an PFTM.

From Properties 1,2 and Corollory 3.1 it is evident that $A \rightarrow_{L}\left(B \rightarrow_{L}\left(A \vee_{L} B\right)\right)$ is an PFTM.

Property 3.6. For any two PFMs $A, B \in \mathrm{P}_{m n}$, then the given statements are PFTMs.

(i) $\left(A \wedge_{L} B\right) \rightarrow_{L} A$.

(ii) $\left(A \wedge_{L} B\right) \rightarrow_{L} B$.

\section{(i) $A \wedge_{L} B$}

$=\left(\left|\sqrt{\max \left(0, a_{i j}^{2}+b_{i j}^{2}-1\right)}, \sqrt{\min \left(1,{a_{i j}^{\prime}}^{2}+b_{i j}^{\prime 2}\right)}\right|\right)$.

$\left(A \wedge_{L} B\right) \rightarrow_{L} A$

$\left.=\left(\mid \sqrt{\max \left(0, a_{i j}^{2}+b_{i j}^{2}-1\right)}, \sqrt{\min \left(1, a_{i j}^{\prime}{ }^{2}+b_{i j}^{\prime 2}\right)}\right)\right) \rightarrow_{L} A$

If $A \wedge_{L} B=(1,0)$ then $\left(A \wedge_{L} B\right) \rightarrow_{L} A=(1,0)$.

Otherwise

$$
\begin{aligned}
\left(A \wedge_{L} B\right) \rightarrow_{L} A & =\left(\mid \sqrt{\min \left(1, a_{i j}^{\prime}{ }^{2}+b_{i j}^{\prime 2}+a_{i j}^{2}\right)},\right. \\
& \left.\sqrt{\max \left(0, a_{i j}^{2}+b_{i j}^{2}+{a_{i j}^{\prime}}^{2}-1-1\right)} \mid\right)
\end{aligned}
$$

Since $\sqrt{\min \left(1, a_{i j}^{\prime 2}+b_{i j}^{\prime 2}+a_{i j}^{2}\right)} \geq$

$\sqrt{\max \left(0, a_{i j}^{2}+b_{i j}^{2}+a_{i j}^{\prime 2}-2\right)}$.

Hence $\left(A \wedge_{L} B\right) \rightarrow_{L} A$ is an PFTM.

(ii) The proof of (ii) is similar to (i).

Property 3.7. If $A \geq B$ then $A \rightarrow_{L} B$ is an PFTM.

Proof:

$A \leq B$ if and only if $a_{i j} \leq b_{i j}$ and $a_{i j}^{\prime} \geq b_{i j}^{\prime}$.

Since $a_{i j}^{\prime} \geq b_{i j}^{\prime}$

$$
\sqrt{{a_{i j}^{\prime}}^{2}+b_{i j}^{2}} \geq \sqrt{b_{i j}^{\prime 2}+a_{i j}^{2}-1} .
$$

Hence $A \rightarrow_{L} B$ is an PFTM.

Property 3.8. For any two PFMs $A, B \in \mathrm{P}_{m n}$, then the given statements are PFTMs.

(i) $\left(A \wedge_{L}\left(A \rightarrow_{L} B\right)\right) \rightarrow_{L} B$.

(ii) $\left(\left(A \rightarrow_{L} B\right) \wedge_{L} B^{C}\right) \rightarrow_{L} A^{C}$.

Proof:

$$
\begin{aligned}
& A \rightarrow_{L} B \\
& =\left(\left|\sqrt{\min \left(1, \quad a_{i j}^{\prime}{ }^{2}+b_{i j}^{2}\right)}, \sqrt{\max \left(0, a_{i j}^{2}+b_{i j}^{\prime 2}-1\right)}\right|\right) .
\end{aligned}
$$

Case (i) If $A \rightarrow_{L} B=(1,0)$ then

$A \wedge_{L}\left(A \rightarrow_{L} B\right)=\left(\left\langle a_{i j}, a_{i j}^{\prime}\right\rangle\right) \wedge_{L}(\langle 0,1\rangle)=\left(\left\langle a_{i j}, a_{i j}^{\prime}\right\rangle\right)=A$.

$\left(A \wedge_{L}\left(A \rightarrow_{L} B\right)\right) \rightarrow_{L} B=\left(A \rightarrow_{L} B\right)=(\langle 0,1\rangle)$.

Case (ii) If $A \rightarrow_{L} B=\sqrt{{a_{i j}^{\prime}}^{2}+b_{i j}^{2}}, \sqrt{b_{i j}^{\prime 2}+a_{i j}^{2}-1}$.

Proof: 


$$
\begin{aligned}
& A \wedge_{L}\left(A \rightarrow_{L} B\right) \\
& =\left(\left\langle a_{i j}, a_{i j}^{\prime}\right\rangle\right) \wedge_{L}\left(\left|\sqrt{a_{i j}^{\prime 2}+b_{i j}^{2}}, \sqrt{b_{i j}^{\prime 2}+a_{i j}^{2}-1}\right|\right) \\
& =\left(\mid \sqrt{\max \left(0, \quad a_{i j}^{2}+a_{i j}^{\prime 2}+b_{i j}^{2}-1\right)},\right. \\
& \left.\left.\sqrt{\min \left(1, a_{i j}^{2}+a_{i j}^{\prime 2}+b_{i j}^{\prime 2}-1\right)}\right\rangle\right)
\end{aligned}
$$

Subcase (ii.a) If $A \wedge_{L}\left(A \rightarrow_{L} B\right)=(\langle 0,1\rangle)$ then

$$
\left(A \wedge_{L}\left(A \rightarrow_{L} B\right)\right) \rightarrow_{L} B=(\langle 0,1\rangle)=\left(\left\langle b_{i j}, b_{i j}^{\prime}\right\rangle\right)=(\langle 0,1\rangle) \text {. }
$$

$$
\begin{aligned}
& \left(\mid \sqrt{\min \left(1, \quad a_{i j}^{\prime}{ }^{2}+a_{i j}^{2}+{b_{i j}^{\prime}}^{2}-1+b_{i j}^{2}\right)},\right. \\
& \left.\sqrt{\max \left(0, a_{i j}^{2}+a_{i j}^{\prime 2}+b_{i j}^{2}-1+b_{i j}^{\prime 2}-1\right)} \mid\right)
\end{aligned}
$$

Now it is clear that

$$
\begin{aligned}
& \left(\mid \sqrt{\min \left(1, \quad a_{i j}^{\prime 2}+a_{i j}^{2}+{b_{i j}^{\prime}}^{2}-1+b_{i j}^{2}\right)}\right. \\
& \left.\geq \sqrt{\max \left(0, a_{i j}^{2}+a_{i j}^{\prime 2}+b_{i j}^{2}-1+b_{i j}^{\prime 2}-1\right)} \mid\right)
\end{aligned}
$$

Hence from case (i) and (ii) $\left(A \wedge_{L}\left(A \rightarrow_{L} B\right)\right) \rightarrow_{L} B$ is an PFTM.

(ii)

$$
\begin{aligned}
& A \rightarrow_{L} B \\
& =\left(\left|\sqrt{\min \left(1, \quad a_{i j}^{\prime 2}+b_{i j}^{2}\right)}, \sqrt{\max \left(0, a_{i j}^{2}+b_{i j}^{\prime 2}-1\right)}\right|\right) . \\
& \left(A \rightarrow_{L} B\right) \wedge_{L} B^{C} \\
& =\left(\left|\sqrt{\min \left(1, \quad a_{i j}^{\prime 2}+b_{i j}^{2}\right)}, \sqrt{\max \left(0, a_{i j}^{2}+b_{i j}^{\prime 2}-1\right)}\right|\right) \\
& \wedge_{L}\left(\left\langle b_{i j}^{\prime}, b_{i j}\right\rangle\right)
\end{aligned}
$$

Case (i) If $\left(A \rightarrow_{L} B\right)=(1,0)$ then

$$
\begin{aligned}
& \left(A \rightarrow_{L} B\right) \wedge_{L} B^{C}=(1,0) \wedge_{L}\left(\left\langle b_{i j}^{\prime}, b_{i j}\right\rangle\right)=\left(\left\langle b_{i j}^{\prime}, b_{i j}\right\rangle\right) \\
= & \left(\left|\sqrt{\min \left(1, \quad b_{i j}^{2}+a_{i j}^{\prime}{ }^{2}\right)}, \sqrt{\max \left(0, b_{i j}^{\prime 2}+a_{i j}^{2}-1\right)}\right\rangle\right) \\
= & \left(A \rightarrow_{L} B\right)=(1,0) .
\end{aligned}
$$

Hence $\left(\left(A \rightarrow_{L} B\right) \wedge_{L} B^{C}\right) \rightarrow_{L} A^{C}$ is an PFTM.

\section{CONCULUSION}

Defind Pythagorean fuzzy tautologial matrices and Pythagorean fuzzy cotautological matrices and some properties of Lukasiwicz implication operator over
Pythagorean fuzzy tautologial matrices and Pythagorean fuzzy cotautological matrices are investigated. Also discussed the relation between implication with Lukasiewicz disjunction and conjunction operations of PFCMs and PFCTMs.

\section{REFERENCES}

[1] K. Atanassov, R. Tcvetkov, On Lukasiewicz's intuitionistic fuzzy disjunction and conjunction. Annual of Informatics Section, Union of Scientists in Bulgaria, 3, (2010), 90-94.

[2] J. Boobalan and S. Sriram., Abraham New operations on intuitionistic fuzzy matrices, International Journal of Pure and Engineering Mathematics, 3(1), (2015), 177-186.

[3] S. K. Khan, M. Pal and A. K. Shyamal, Intuitionistic fuzzy matrices, Notes on Intuitionistic Fuzzy Sets, 8(2), (2002), 51-62.

[4] S. K. Khan and M. Pal, Intuitionistic fuzzy Tautological Matrices, V.U Journal of physical Sciences, 8 (2002-2003), 92 100.

[5] P. Murugadas and K. Lalitha, Intuitionistic Fuzzy Cotautological Matrices, International Journal of Pure and Applied Mathematics, 103 (3), (2015), 453-466.

[6] T. Muthuraji and S. Sriram, Commutative monoids and monoid homomorphism on Lukasiewicz conjunction and disjunction operators over intuitionistic fuzzy matrices, International Journal of Pure and Engineering Mathematics, 3(2), (2015), 63-75.

[7] T. Muthuraji, Some Properties of Operations Conjunction, Disjunction and Implication operators on intuitionistic fuzzy matrices, Journal of Advances in Mathematics, 11(3), (2019), 5000-5006.

[8] T. Muthuraji, Some Properties of Operations Conjunction, Disjunction and Implication from Lukasiewicz's type over intuitionistic fuzzy matrices, AIP Conference Proceedings, 2177(020052), (2019), 1-6.

[9] T. Muthuraji and B. Anitha, Lukasiewicz imlication on intuitionistic fuzzy tautological matrices, Journal of Emerging Technology and innavative Research, 6(4), (2019), 1-6.

[10] M. Pal, Intuitionistic fuzzy determinant, Vidyasagar University Journal of Physical Sciences, 7, (2001), 87-93.

[11] I. Silambarasan and S. Sriram, Algebraic operations on Pythagorean fuzzy matrices, Mathematical Sciences International Journal, 7(2), (2018), 406-414.

[12] D. Venkatesan and S. Sriram, Multiplicative operations of Pythagorean Fuzzy Matrices, Malaya Journal of Matematik, S(1), (2019), 389-393.

[13] D.Venkatesan and S. Sriram, Commutative monoids and monoid homomorphism on Lukasiwicz conjunction and disjunction operations over Pythagorean fuzzy matrices, Indian Journal of Science and Technology, 12(10), (2015), 1-9.

[14] D.VenkatesanandS.Sriram,OnLuckasiewiczDisjunctionandConj unctionofPythagoreanFuzzyMatries, International Journal of Computer Sciences and Engineering, 7(6), (2019), 861-865.

[15] R. R. Yager and A. M. Abbasov, Pythagorean membership grades, complex numbers, and decision making, International Journal of Intelligence Fuzzy Systems, 28(5), (2013), 436-452.

[16] X.. L. Zhang and Z. S. Xu, Extension of TOPSIS to multiple criteria decision making with Pythagorean fuzzy sets, International Journal of Intelligence Systems, 29(12), (2014), 1061-1078 